\title{
Calorimetric examination of hip pseudo-capsule after secondary hip surgeries
}

\author{
G. Búcs ${ }^{1} \cdot$ L. G. Nöt ${ }^{1} \cdot$ Á. Dandé ${ }^{1} \cdot$ L. Kereskai ${ }^{2} \cdot$ D. Lörinczy ${ }^{3}$ \\ Received: 18 November 2018/Accepted: 19 February 2019/Published online: 5 March 2019 \\ (C) The Author(s) 2019
}

\begin{abstract}
During revision hip surgeries, a soft pseudo-capsule is formed around the artificial joint. The roles of the pseudo-capsule are to protect and to stabilize the joint. Based on intraoperative observation, we have found that different types of pseudocapsules have different thickness and elasticity, compared to the primary joint capsule. The purpose of the study was to evaluate and identify the different types of pseudo-capsules, based on thermal characteristics and histological examinations. The pseudo-capsule samples were collected during aseptic hip revision $(n=3)$, septic hip revision $(n=2)$ and during revision surgery after antibiotic-impregnated spacer implantation $(n=3)$. The thermal characteristics (maximal denaturation temperature, $T_{\mathrm{m}}$, and calorimetric enthalpy change, $\left.\Delta H_{\mathrm{cal}}\right)$ were determined from the measurements made by SETARAM Micro DSC-II calorimeter between $37^{\circ}$ and $90^{\circ} \mathrm{C}$ with $0.3 \mathrm{~K} \mathrm{~min}^{-1}$ heating rate. The denaturation scans of different pseudo-capsule samples have clearly demonstrated specific, representative changes, comparing samples with aseptic and septic origins. These changes were confirmed by histological evaluations; therefore, DSC could be a useful tool in the evaluation of hip pseudo-capsules with different origins, providing further help in the differential diagnostics of septic conditions.
\end{abstract}

Keywords Hip replacement $\cdot$ Revision $\cdot$ Pseudo-capsule $\cdot$ Sepsis $\cdot$ Collagen $\cdot$ Calorimetry

\section{Introduction}

During the recent decades, there has been a continuous worldwide growth in the number of hip replacements. By the twenty-first century, due to the developments in surgical techniques, hip replacements based on both orthopedic and trauma indications became safer and available for more patients. The surgical procedures utilize more types of prosthesis and, importantly, better surgical approaches [1-3].

\section{Lőrinczy}

denes.lorinczy@aok.pte.hu

1 Department of Traumatology and Surgery, Clinical Center, Medical School, University of Pécs, Ifjúság str. 13, Pécs 7624, Hungary

2 Department of Pathology, Medical School, University of Pécs, Szigeti str. 12, Pécs 7624, Hungary

3 Department of Biophysics, Medical School, University of Pécs, Szigeti str. 12, Pécs 7624, Hungary
The different types of surgical approaches allow preserving the joint capsule (capsulotomy), instead of removal (capsulectomy) during hip arthroplasties. The preservation of joint capsule could provide more primary stability, therefore further decreasing the rate of hip dislocations during the postoperative 6-12 weeks.

Months or years after the primary hip replacements utilizing capsulectomy, a pseudo-capsule develops around the implants. The pseudo-capsule found during secondary hip surgeries has different characteristics, compared to the primary joint capsule [4-6]. Furthermore, the pseudomembrane macroscopically seems to be scar tissue, but the elasticity and thickness may be different in case of aseptic and septic hip conditions.

The differential scanning calorimetry has been found useful in the differential diagnosis of various musculoskeletal diseases, including degenerative, inflammatory and septic conditions [7-13].

Therefore, the purpose of this study was to evaluate and identify the different types of pseudo-capsules, based on thermal characteristics and histological examinations. 


\section{Materials and methods}

\section{Sample collection}

The $4 \times 4 \times 20$-mm-sized sample of the anterior part of the pseudo-capsule was resected during secondary hip replacements, which was performed using DAA (direct anterior approach). Then, samples were properly stored for further measurements, as described in details elsewhere [14]. The pseudo-capsule samples were obtained from patients who underwent revision surgery: (1) due to aseptic loosening ( $n=3)$, (2) due to septic loosening $(n=2)$ or (3) after antibiotic-impregnated spacer insertion $(n=3)$. These samples were considered as waste from the point of surgery. In case of septic loosening, increased levels of inflammatory parameters, such as leukocytes and CRP (Creactive proteins), were detected. In each of the cases, the presence of bacteria was confirmed by microbiological culturing, samples collected from multiple points from the surface of the removed implant and the medullar cavity.

The study protocol has been prepared in accordance with the ethical guidelines of the 2003 Declaration of Helsinki. After receiving permission from the Local Ethics Committee, patients or nearest relatives provided a signed informed consent after they were informed clearly about details of the study and joint capsule sampling.

\section{Histological processing}

The samples from the pseudo-capsules were fixed in $4 \%$ formaldehyde for at least 2 days. After fixation, two representative sections from each sample were cut. Then, the tissues were dehydrated and embedded in paraffin according to the standard histopathological processing and $4-\mu$ m-thick sections were cut by microtome. The slides were stained with hematoxylin-eosin.

\section{Thermal analysis}

We have investigated the thermal denaturation of pseudocapsule samples by a SETARAM Micro DSC-II calorimeter. The analysis was made between 0 and $100{ }^{\circ} \mathrm{C}$ with a heating rate of $0.3 \mathrm{~K} \mathrm{~min}^{-1}$. Conventional Hastelloy batch vessels $\left(V_{\max }=1 \mathrm{~mL}\right)$ were used for the experiment. The average sample size was $4 \times 4 \times 20 \mathrm{~mm}$. Normal saline was used as a reference. The reference and sample vessels were equilibrated with a precision of $\pm 0.1 \mathrm{mg}$; this way we did not have to do any correction between vessels' heat capacity. With the help of a two-point setting SETARAM peak integration, calorimetric enthalpy change was calculated from the area under the heat absorption curve; then, the results (denaturation or melting temperature $\left(T_{\mathrm{m}}=\right.$ temperature, where $50 \%$ of the heated sample will be denatured) and calorimetric enthalpy change $\left(\Delta \mathrm{H}_{\mathrm{cal}}\right)$ data of samples) were compared.

\section{Results}

\section{Histological evaluation}

The main characteristics of the samples were analyzed, such as collagen and fat ratio, absence or presence and severity of inflammation, absence or presence of destructed bone fragments and additional changes.

The samples from the patients with aseptic loosening were invariably cell-poor collagen-rich tissues without the presence of inflammation. In some cases, embedded bone fragments or pigment was seen (Fig. 1).

The samples from the patients with septic loosening have shown moderate hyper-cellularity with fibroblasts, macrophages and focal hyper-cellular inflammatory areas which located frequently in the perivascular area. In these foci and in the interstitium, mainly lymphocytes, macrophages and scattered neutrophils are seen (Fig. 2a-b).

In the histological samples from those patients who underwent antibiotic-impregnated spacer insertion again, mainly cell-poor collagenous tissue is visible; in one sample, increased fat content was detected. No significant inflammation was exhibited (Fig. 3).

\section{DSC measurements}

The result of denaturation of hip pseudo-capsules is shown in Fig. 4. The obtained curves show that the half width $\left(T_{1 / 2}\right)$ of transitions is decreasing in case if the antibioticimpregnated spacer was implanted. These changes indicate a more cooperative structure of the pseudo-capsule, likely due to the preventive effect of the constantly released antibiotic. The $T_{1 / 2}$ decreases further in septic loosening, probably as a consequence of structural changes in response to perivascular inflammation.

To compare the effects of aseptic and septic loosening on the structural changes of pseudo-capsule, the

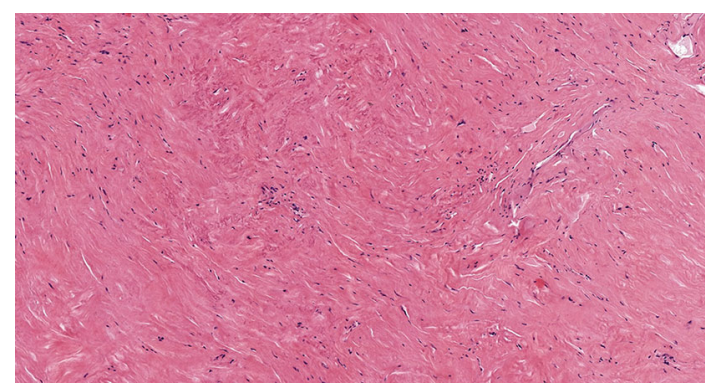

Fig. 1 Histological image of pseudo-capsule in aseptic revision surgery (stained with hematoxylin-eosin) 


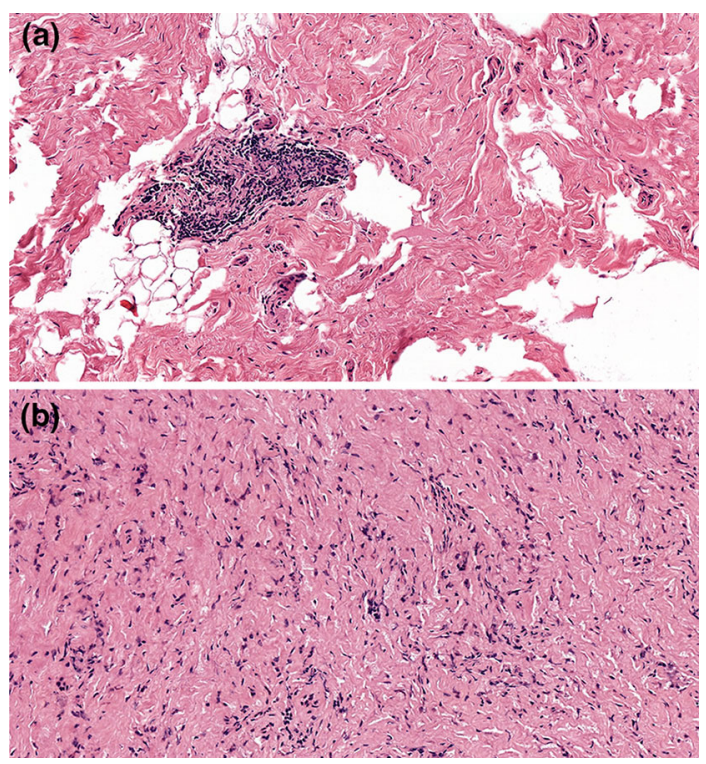

Fig. 2 Histological image: a pseudo-capsule in septic revision surgery with perivascular inflammation and $\mathbf{b}$ pseudo-capsule in aseptic revision surgery with hyper-cellularity (staining as in Fig. 1)

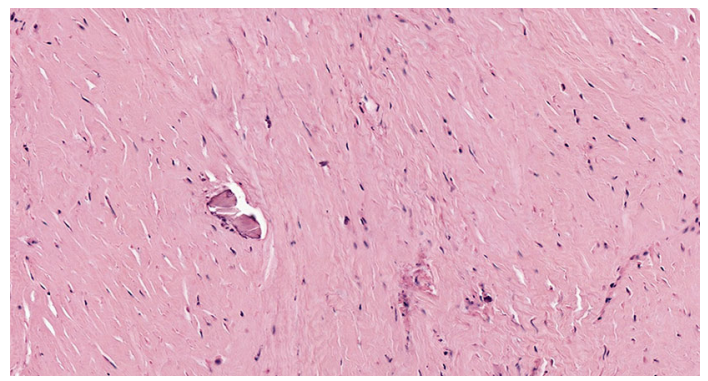

Fig. 3 Pseudo-capsule in revision surgery with antibiotic spacer (staining as in Fig. 1)

calorimetric enthalpy seems to be the practical measure of the severity of problem.

The results shown in Table 1 clearly demonstrate the dependence of denaturation temperature and calorimetric enthalpy on the severity of loosening. Applying antibioticimpregnated spacer, the decrease in $\Delta H_{\text {cal }}$ is about $20 \%$, while in case of septic loosening it is $\sim 50 \%$, indicating extended loosening of the structure of pseudo-capsules. The number of collected samples is not enough to make verified statistical conclusion, but based on thermal parameters the severity of different types of loosening can be well separated.

\section{Discussion}

We have previously shown that differential scanning calorimetry provides useful data in the analyses of capsules from patients with different types of hip diseases. The denaturation curves indicated a significantly different,

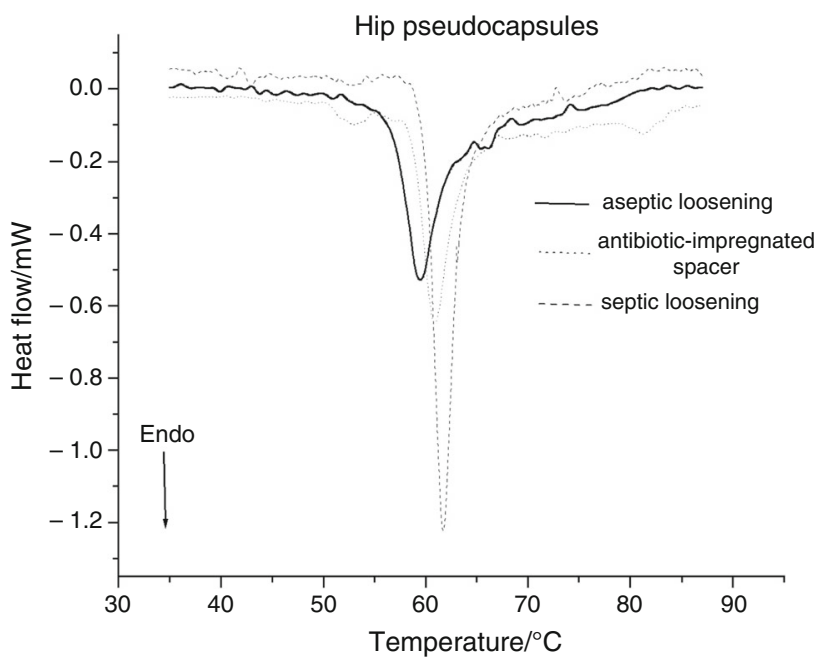

Fig. 4 DSC scans of hip pseudo-capsules. Symbols: solid line: aseptic loosening, dotted line: effect of antibiotic-impregnated spacer and dashed line: septic loosening. The curves are averages (Table 1), and endotherm deflection is directed downwards

Table 1 Maximal denaturation temperature $\left(T_{\mathrm{m}}\right)$ and mass-normalized calorimetric enthalpy $\left(\Delta H_{\mathrm{cal}}\right)$ of hip pseudo-capsules (data show average values, rounded to two places)

\begin{tabular}{lll}
\hline Sample & \multicolumn{2}{l}{ Thermal parameters } \\
\cline { 2 - 3 } & $T_{\mathrm{m}} /{ }^{\circ} \mathrm{C}$ & $\Delta \mathrm{H}_{\text {cal }} / \mathrm{J} \mathrm{g}^{-1}$ \\
\hline Aseptic loosening $(n=3)$ & 60.85 & 4.28 \\
Antibiotic-impregnated spacer $(n=3)$ & 60.02 & 3.40 \\
Septic loosening $(n=2)$ & 61.53 & 2.33 \\
\hline
\end{tabular}

individual pattern for each type of degenerative or inflammatory hip diseases [13]. Recently, Dande et al. have demonstrated that thermal analyses of synovial fluid of the knee joint could help in the diagnostics of septic conditions [14].

In case of revision hip arthroplasties, detection and confirmation of bacterial presence can be difficult. The patients' symptoms, the time elapsed from the primary surgery, results of imaging and laboratory investigations may contribute only in part to reach the right diagnosis. Moreover, the intraoperative diagnosis of joint infections could also be challenging and the microbiological confirmation of bacterial presence may take days, occasionally weeks $[15,16]$.

Here, we found that the thermal denaturation scans of different pseudo-capsule samples have clearly demonstrated specific, representative changes, comparing samples with aseptic to septic origins. It could be explained by the conformational changes of the tissue collagens in response to bacterial toxins and the release of inflammatory 
mediators in septic conditions [7, 9]. Moreover, these changes were confirmed by histological evaluations as well.

Meanwhile, the pseudo-capsule samples collected during aseptic revision surgeries or after gentamycin-impregnated spacer implantation showed similar thermoanalytical characteristics and histological results, in both cases due to the absence of bacterial infection.

This study has a potential limitation because of the relatively small number of samples; therefore, we did not perform standard deviation calculation (and this way statistical analysis) because the number of samples $(n)$ was smaller, than five. The mean values were analyzed to evaluate the effect of septic conditions on the measured thermal parameters. However, despite this limitation, we believe that the differences observed in the thermal characteristics and the shape of DSC scans provide valuable and scientifically relevant data in the analysis of hip pseudo-capsules.

\section{Conclusions}

DSC could be a useful tool in the evaluation of hip pseudocapsules with different origins, providing further help in the differential diagnostics of septic complications.

Acknowledgements Open access funding provided by University of Pécs (PTE). This work was supported by Grant OTKA CO-272 (for D. Lörinczy).

Open Access This article is distributed under the terms of the Creative Commons Attribution 4.0 International License (http://creative commons.org/licenses/by/4.0/), which permits unrestricted use, distribution, and reproduction in any medium, provided you give appropriate credit to the original author(s) and the source, provide a link to the Creative Commons license, and indicate if changes were made.

\section{References}

1. Pearson MJ, Grover LM, Lord JM, Jones SW, Davis ET. Bearings in hip arthroplasty: joint registries vs precision medicine: review article. HSS J. 2017;13(1):20-7.

2. Coomber R, Porteous M, Hubble MJ, Parker MJ. Total hip replacement for hip fracture: surgical techniques and concepts. Injury. 2016;47(10):2060-4.

3. Tol MC, van den Bekerom MP, Sierevelt IN, Hilverdink EF, Raaymakers EL, Goslings JC. Hemiarthroplasty or total hip arthroplasty for the treatment of a displaced intracapsular fracture in active elderly patients: 12-year follow-up of randomised trial. Bone Jt J. 2017;99-B(2):250-4.

4. Munoz-Mahamud E, Soriano A, Combalia A, Medrano C, Bosch $\mathrm{J}$, Garcia S, et al. Comparison of bacterial results from conventional cultures of the periprosthetic membrane and the synovial or pseudocapsule during hip revision arthroplasty. Arch Orthop Trauma Surg. 2014;134(4):577-83.

5. Bori G, Muñoz-Mahamud E, Garcia S, Mallofre C, Gallart X, Bosch J, et al. Interface membrane is the best sample for histological study to diagnose prosthetic joint infection. Mod Pathol. 2011;24(4):579-84.

6. Miki H, Masuhara K. Arthrographic examination of the pseudocapsule of the hip after posterior dislocation of total hip arthroplasty. Int Orthop. 2000;24(5):256-9.

7. Wiegand N, Naumov I, Vámhidy L, Kereskai L, Lőrinczy D, Nöt LG. Comparative calorimetric analysis of 13 different types of human healthy and pathologic collagen tissues. Thermochim Acta. 2013;568:171-4.

8. Naumov I, Wiegand N, Patczai B, Vámhidy L, Lőrinczy D. Differential scanning calorimetric examination of the human hyaline cartilage of the femoral head after femoral neck fracture. J Therm Anal Calorim. 2012;108(1):59-65.

9. Nöt LG, Naumov I, Vámhidy L, Lörinczy D, Wiegand N. Comparison of thermal characteristics of degenerated and inflamed human collagen structures with differential scanning calorimetry. J Therm Anal Calorim. 2013;113(1):273-9.

10. Könczöl F, Wiegand N, Nöt LG, Lörinczy D. Examination of the cyclophosphamide-induced polyneuropathy on guinea pig sciatic nerve and gastrocnemius muscle with differential scanning calorimetry. J Therm Anal Calorim. 2014;115(3):2239-43.

11. Patczai B, Mintál T, Nőt LG, Wiegand N, Lőrinczy D. Effects of deep-freezing and storage time on human femoral cartilage. J Therm Anal Calorim. 2017;127(2):1177-80.

12. Wiegand N, Vámhidy L, Lörinczy D. Differential scanning calorimetric examination of ruptured lower limb tendons in human. J Therm Anal Calorim. 2010;101(2):487-92.

13. Bücs G, Nöt LG, Dandé Á, Wiegand N, Lőrinczy D. Detection of joint capsule changes by differential scanning calorimetry (DSC) in different types of hip disorders to evaluate surgical techniques (a preliminary report). J Therm Anal Calorim. 2017;130(3):1293-6.

14. Dandé Á, Nöt LG, Wiegand N, Kocsis B, Lőrinczy D. DSC analysis of human synovial fluid samples in the diagnostics of non-septic and septic arthritis. J Therm Anal Calorim. 2017;130(3):1249-52.

15. Dandé Á, Nőt LG, Bűcs G, Wiegand N, Kocsis B, Lőrinczy D. Examination of typical bacterial strains in septic arthritis by isoperibol calorimeter. $\mathrm{J}$ Therm Anal Calorim. 2018;131(3):2041-8.

16. Naumov I, Lörinczy D, Vámhidy L, Than P, Wiegand N. Differential scanning calorimetric examination of the interfacial membrane in failed hip joint replacements. J Therm Anal Calorim. 2012;109(2):783-7.

Publisher's Note Springer Nature remains neutral with regard to jurisdictional claims in published maps and institutional affiliations. 